\title{
ON A FAMILY OF WEIGHTED CONVOLUTION ALGEBRAS
}

\author{
HANS G. FEICHTINGER and A. TURAN GÜRKANLI (1)
}

\author{
Institut für Mathematik \\ Universität Wien \\ Strudlhofgasse 4 \\ A-1090 Wien, Austria
}

\author{
Ondokuz Mayis University \\ Faculty of Art and Sciences \\ Department of Mathematics \\ Samsun, Turkey
}

(Received March 7, 1988)

ABSTRACT. Continuing a line of research initiated by Larsen, Liu and Wang [12], Martin and Yap [13], Gürkanli [5], and influenced by Reiter's presentation of Beurling and Segal algebras in Reiter [2,10] this paper presents the study of a family of Banach ideals of Beurling algebras $L_{w}^{1}(G), G$ a locally compact Abelian group. These spaces are defined by weighted $\mathrm{L}^{\mathrm{P}}$-conditions of their Fourier transforms. In the first section invariance properties and asymptotic estimates for the translation and modulation operators are given. Using these it is possible to characterize inclusions in section 3 and to show that two spaces of this type coincide if and only if their parameters are equal. In section 4 the existence of approximate identities in these algebras is established, from which, among other consequences, the bijection between the closed ideals of these algebras and those of the corresponding Beurling algebra is derived.

KEY WORDS AND PHRASES. Beurling algebra, weighted ${ }^{\mathrm{p}}$-spaces, convolution, ideal theorem, Banach ideals, factorization 1980 AMS SUBJECT CLASSIFICATION CODES. 43A15

\footnotetext{
${ }^{1}$ Acknowledgement: Supported by the Scientific and Technical Research Council of Turkey and the Foundation of the Ondokuz Mayis University

2 Due to a Max Kade fellowship the first named author spends the academic year 1989/90 at the University of Maryland at College Park, MD, 20742, which allowed him to complete the manuscript there.
} 


\section{NOTATIONS}

Throughout G denotes a locally compact group G with dual group $\hat{G}$, and Haar measure $\mathrm{dx}$ and $\hat{\mathrm{dx}}$ respectively. In order to avoid trivialites we assume throughout to have non-discrete and non-compact groups. We write $\mathcal{K}(G)$ for the space of all continuous, complex-valued functions on $G$ with compact support. For $1 \leq p \leq \infty$ we write $\left(L^{p}(G), \|_{p}\right)$ the Lebesgue spaces .

A BF-space on $G$ is a Banach space $\left(B,\|\|_{B}\right)$ of (classes of) measurable functions embedded into $\mathrm{L}_{l o c}^{1}(G)$, i.e.such that for any compact set $K \leqslant G$ there exists some constant $C_{K}>0$ with $\left\|f c_{K}\right\|_{1} \leq C_{K}\left\|_{B}\right\|_{B}$ for all $f \in B$ (where $c_{K}$ is the characteristic function of $K$ ). The translation operators $L_{y}$ are given by $L_{y} f(x):=f(x-y)$, and the multiplication operator ${ }^{M}$ is defined as $M_{\chi} f(x):=\chi(x) f(x)$ for $x, y \in G$ and $\chi \in \hat{G} .\left(B,\|\|_{B}\right)$ is called [strongly] translation invariant if one has $L_{y} B \subseteq B$ [and $\left\|L_{y} f\right\|_{B}=\|f\|_{B}$ ] for all $f \in B$ and $y \in G$. [Strong] Character invariance is defined in the same way.

A Banach space $\left(B,\|\|_{B}\right)$ is called a Banach module over a Banach algebra $\left(A,\|\|_{A}\right)$ if $B$ is a module over $A$ in the algebraic sense for some multiplication, $(a, b) \rightarrow a \cdot b$, and satisfies $\|a \cdot b\|_{B} \leq\|a\|_{A}\|b\|_{B}$. If $B \hookrightarrow A$, i.e. if $B$ is continuously embedded into $A$ and the multiplication in $A$ satisfies the above estimate, we call $B$ a Banach ideal in A. A net (e $\left.{ }_{\alpha}\right)_{\alpha \in I}$ in $A$ is called a (bounded) approximate identity for $A$ (shortly BAI), if one has $\quad \lim _{\alpha}\left\|e_{\alpha} a-a\right\|_{A} \rightarrow 0$ for any $a \in A$. A Banach module (ideal) is called essential if the closed linear span of $A \cdot B$ in $\left(B,\|\|_{B}\right)$ coincides with B. If $A$ has a BAI $\left(e_{\alpha}\right)_{\alpha \in I}$ this is equivalent (Braun and Feichtinger [1]) to assume that one has $\lim _{\alpha}\left\|e_{\alpha} b-b\right\|_{B} \rightarrow 0$ for all $f \in B$.

For a Beurling weight $w$ on $G$ (Reiter [2]), i.e. a continuous function $w$ satisfying $w(x) \geq 1$ and $w(x+y) \leq w(x) w(y)$ for all $x, y \in G$, we set for $1 \leq \mathrm{p}<\infty$ :

${ }_{L}^{p}(G):=\left\{f \mid f w \in L^{p}(G)\right\}$. It is a Banach space under the natural norm

$$
\|f\|_{p, w}:=\left[\int_{G}|f(x)|^{p_{w}}{ }^{p}(x) d x\right]^{1 / p} \text {. }
$$

Recall that one has $L_{w_{1}}^{p}(G) \subseteq L_{w_{2}}^{p}(G)$ if and only if $w_{2}\left(w_{1}\right.$, i.e. $w_{2}(x) \leq C w_{1}(x)$ for all $x \in G$ (Feichtinger [3]). Two positive functions are called equivalent, we write $w_{1} \approx w_{2}$, if $w_{2}\left(w_{1}\right.$ and $w_{1}\left(w_{2} \cdot L_{w}^{1}(G)\right.$ is called a Beurling algebra, because it is a Banach algebra with respect to convolution. It always has a BAI. Moreover ${ }_{L_{w}}^{p}(G)$ is an essential Banach module over $L_{w}^{1}(G)$ with respect to convolution for $1 \leq p<\infty$ (Braun and Feichtinger [1] Prop.1, actually, it follows by writing convolution as vector-valued integral).

For functions in $L^{1}(G)$ the Fourier transform is denoted alternatively by $\hat{f}$ or If (if we want to stress the Fourier transform $f \longmapsto \hat{f}=$ Ff as a mapping). Given a normed space $\left(B,\|\|_{B}\right) \hookrightarrow L^{1}(G)$ we consider $F B$ always as the image of $B$ under $\mathscr{F}$, endowed with its natural norm $\left\|F_{f}\right\|_{\mathscr{F} B}:=\|f\|_{B}$. For any subspace $I \subseteq L^{1}(G)$ we define the set cosp(I) by

$$
\operatorname{cosp}(I):=\{x \in \hat{G}, \hat{f}(\chi)=0 \text { for all } \mathrm{f} \in I\} \text {. }
$$


2. BASIC RESULTS AND INVARIANCE PROPERTIES.

Let $w, \omega$ be weight functions on $G$ and $\hat{G}$ respectively. For $1 \leq p<\infty$ we set $A_{w, \omega}^{p}(G):=\left\{f \mid f \in L_{w}^{1}(G), \hat{f} \in L_{w}^{p}(\hat{G})\right\}$, and $\|f\|_{w, \omega}^{p}:=\|f\|_{1, w}+\|\hat{f}\|_{p, \omega}$.

Among the most natural examples for spaces of this type (describing both decay properties of its elements plus a certain amount of smoothness) are the spaces $L_{w}^{1} n \varphi_{s}^{2}\left(\mathbb{R}^{m}\right)$ for $s>0$, where $\mathscr{L}_{s}^{2}\left(\mathbb{R}^{m}\right)$ denotes the Bessel potential spaces of order $s$ (Stein [4]). In fact, these spaces arise as spaces $A_{w, \omega}^{p}\left(\mathbb{R}^{m}\right)$ for $\omega(y):=\left(1+|y|^{2}\right)^{s / 2}$. For special cases see Gürkanli [5].

The basic observation concerning $A_{W, \omega}^{p}$-spaces is the following one:

THEOREM 2.1. $\left(A_{w, \omega}^{p}(G),\|\|_{w, \omega}^{p}\right)$ is a Banach ideal in $L_{w}^{1}(G)$, hence a Banach algebra with respect to convolution.

PROOF. The result can be obtained either by slight modifications of the proof of Thm.3.1 in Feichtinger [6] or of Theorems 1,2 in Gürkanli [5].

LEMMA 2.2. For any $f \in L_{w}^{p}(G), f \neq 0$, the function $x \rightarrow\left\|L_{x} f\right\|_{p, w}$ is equivalent to the weight function $w$, i.e. there is a constant $C>0$ such that one has

$$
C^{-1} w(x) \leq\left\|L_{x} f\right\|_{p, w} \leq C \cdot w(x) \text { for all } x \in G \text {. }
$$

PROOF. For the second estimate we write for $f \in L_{W}^{p}(G)$

$$
\left\|L_{x} f\right\|_{p, w}=\left(s|f(z-x)|^{p_{w}} p_{(z) d z}\right)^{1 / p}=\left(s|f(u)|^{p} p^{p}(x+u) d u\right)^{1 / p} \leq w(x)\|f\|_{p, w} .
$$

For the first estimate choose a compact set $K \subseteq G$ with $\left\|f \cdot c_{K}\right\|_{p, w}>0$. Then $\left\|L_{x} f\right\|_{p, w} \geq\left(s_{x+K}|f(z-x)|^{p_{w}} p_{(z) d z}\right)^{1 / p}=\left(\int_{K}|f(u)|^{p_{w}} p_{(x+u) d u}\right)^{1 / p} \geq$ $\geq\left\|f \cdot c_{K}\right\|_{p} w(x) / \sup _{u \in K} w(-u)$. Since $w$ is locally bounded (cf. [6]) the proof is finished by setting $C:=\max \left\{\|f\|_{p, w}, w(x) / \sup _{u \in K} w(-u)\right\}$.

LEMMA 2.3. For any $f \in A_{W, \omega}^{p}(G), f \neq 0$, the function $x \longmapsto\left\|{ }_{\chi} f\right\|_{W, \omega}^{p}$ is equivalent to the weight function $\omega$.

PROOF. The proof is similar to that of Lemma 2.2 and is left to the interested reader.

THEOREM 2.4. $\quad \mathrm{A}_{\mathrm{W}, \omega}^{\mathrm{p}}(\mathrm{G})$ is translation and character invariant. Moreover, the functions $\quad \mathrm{W} \longmapsto\left\|\mathrm{L}_{x}\right\|_{W, \omega}^{\mathrm{p}}$ and $\chi \longmapsto\left\|_{\chi}\right\|_{W, \omega}^{p}$ are equivalent to the weight functions $W$ and $\omega$ respectively.

PROOF. Combining Lemma 2.2 and Lemma 2.3 gives the result, by observing that for the lower estimate any expression with $f$ with $\|f\|_{w, \omega}=1$ is appropriate.

3. INCLUSIONS AND CONSEQUENCES.

We start with the observation that the intersection of two weighted ${ }_{L}^{p}$-spaces ${ }_{L}^{p}{ }_{w_{1}}^{p}(G)$ and $L_{w_{2}}^{p}(G)$ is just $L_{w}^{p}(G)$, where $w$ may be taken as $w=\max \left(w_{1}, w_{2}\right)$. Hence we have $A_{w_{1}, w_{1}}^{p_{1}} \cap A_{w_{2}, w_{2}}^{p_{2}}=A_{w, w}^{p}$, with $w=\max \left(w_{1}, w_{2}\right)$, and $\omega=\max \left(\omega_{1}, \omega_{2}\right)$. Next we look for inclusions. 
LEMMA 3.1. Inclusions between $A_{W, \omega}^{p}(G)$-spaces are automatically continuous, i.e. given weight functions $w_{1}, w_{2}, \omega_{1}, \omega_{2}$ on $G$ and $\hat{G}$ respectively one has: If $A_{W_{1}, \omega_{1}}^{p_{1}}(G) \subseteq A_{W_{2}, \omega_{2}}^{p_{2}}(G)$ then there is some constant $C>0$ such that $\|f\|_{W_{1}, \omega_{2}}^{p_{1}} \leq C \cdot\|f\|_{W_{2}, \omega_{2}}^{p_{2}}$ for all $f \in A_{w_{1}, \omega_{1}}^{p_{1}}$ (G).

PROOF. The result follows from the fact, that the $A_{W, \omega}^{p}(G)$-spaces are Banach spaces, continuously embedded into $L^{1}(G)$. Thus the closed graph theorem can be applied in order to check that the inclusion mapping has closed graph, hence is continuous.

THEOREM 3.3. $A_{w_{1}, \omega_{1}}^{p}$ (G) $\subseteq A_{w_{2}, \omega_{2}}^{p}$ (G) if and only if $w_{2}\left(w_{1}\right.$ and $\omega_{2}\left\{\omega_{1}\right.$.

PROOF. It is obvious that $w_{2}\left(w_{1}\right.$ and $\omega_{2}\left(\omega_{1}\right.$ implies $L_{w_{1}}^{1}(G) \hookrightarrow L_{w_{2}}^{1}(G)$ and $\mathrm{L}_{\omega_{1}}^{\mathrm{p}}(\hat{\mathrm{G}}) \hookrightarrow \mathrm{L}_{\omega_{2}}^{\mathrm{p}}(\hat{\mathrm{G}})$, hence $\mathrm{A}_{\mathrm{w}_{1}, \omega_{1}}^{\mathrm{p}}(\mathrm{G}) \hookrightarrow \mathrm{A}_{\mathrm{w}_{2}, \omega_{2}}^{\mathrm{p}}(\mathrm{G})$.

The converse implication follows by means of Lemma 3.1 with Lemmas 2.2 and 2.3. (using the estimate $\left\|L_{x} f\right\|_{w_{2}, \omega_{2}}^{p} \leq C \cdot\left\|L_{x} f\right\|_{w_{1}, \omega_{1}}^{p}$ ).

COROLLARY 3.3. Two $A_{W, \omega}^{p}$-spaces are equal if and only if the corresponding weights are equivalent.

COROLLARY 3.4. There is an equivalent, strongly character invariant norm for $A_{W, \omega}^{p}(G)$ if and only if $\omega \approx 1$.

PROOF. If $\omega \approx 1$ then $\|\mathrm{f}\|:=\|\mathrm{f}\|_{1, w}+\|\hat{f}\|_{\mathrm{p}}$ is an equivalent strongly character invariant norm on $A_{w, \omega}^{p}(G)$. Conversely, if such an equivalent norm exists it is clear that the function $\chi \longmapsto\left\|M \chi^{\|}\right\|$is bounded on $A_{W, \omega}^{p}(G)$, which implies the boundedness of $\omega$ by Thm. 2.4. since any bounded Beurling weight is equivalent to the constant (e.g. the trivial weight $w(x)=1$ ) weight, the assertion is verified.

COROLLARY 3.5. There is an equivalent, strongly translation invariant norm for $A_{W, \omega}^{\mathrm{p}}(\mathrm{G})$ if and only if $w \approx 1$.

Since the proof is similar to that of 3.4 it is left to the reader.

So far we have compared algebras $A_{W, \omega}^{p}(G)$ with fixed $p$. In order to deal with the situation of different values $p$ and $q$ we have to introduce a slight extra condition: $A$ weight $w$ is said to satisfy the Beurling-Domar condition (shortly: (BD), Domar [7] or Reiter [2]), if one has

$$
\sum_{n \geq 1} n^{-2} \cdot \log \left(w\left(x^{n}\right)\right)<\infty \text { for all } x \in G \text {. }
$$

THEOREM 3.6. Assume that $w$ satisfies (BD) and that $\omega(\chi) \rightarrow \infty$ for $x \rightarrow \infty$ in $\hat{G}$. Then $A_{w, \omega}^{p}(G) \subseteq A_{W, \omega}^{q}(G)$ if and only if $p \leq q$.

PROOF. By Lemma 3.1. we may assume $\|f\|_{W, \omega}^{p} \leq C \cdot\|f\|_{W, \omega}^{q}$ for $f \in A_{W, \omega}^{p}(G)$. Since $\omega(t) \rightarrow \infty$ for $t \rightarrow \infty$ it is possible to find for any $n \geq 1$ some compact set $K_{n} \subseteq \hat{G}$ such that $\omega(t) \geq n^{2}$ for all $t \in K_{n}$. Since $w$ satisfies the (BD)-condition we can find some $f_{0} \in L_{w}^{1}(G)$ such that $\hat{f}_{0}$ has compact support $K_{0}, \hat{f}_{o}(0)=1$. Choosing by induction a sequence $\left(t_{k}\right)_{k=1}^{n}$ in $\hat{G} \backslash K_{n}$ such that $\left(t_{i}+K_{0}\right) \cap\left(t_{j}+K_{o}\right)=\varnothing$ for $i \neq j$. We then define $f_{n}$ by

$$
f_{n}=\sum_{k=1}^{n} \omega^{-1}\left(t_{k}\right) \cdot M_{t_{k}} f_{0}
$$


Therefore

$$
\hat{f}_{n}=\sum_{k=1}^{n} \omega^{-1}\left(t_{k}\right) \cdot L_{t_{k}} \hat{f}_{0}
$$

In order to estimate $\left\|\hat{f}_{n}\right\|_{p, \omega}$ from below we observe first that by the argument used in the second part of the proof of Lemma 2.2 one has $\left\|L_{t_{k}} \hat{f}_{o}\right\|_{p, \omega} \geq c_{o} \cdot \omega\left(t_{k}\right) \cdot\left\|f_{o}\right\|_{p}$ for all $k \geq 1$.

Since these functions have disjoint supports we have

$$
\left(\left\|\hat{f}_{n}\right\|_{p, \omega}\right)^{p}=\sum_{k=1}^{n} \omega^{-1}\left(t_{k}\right) \cdot\left(\left\|L_{t_{k}} \hat{f}_{o}\right\|_{p, \omega}\right)^{p} \geq c_{0} \cdot \sum_{k=1}^{n}\left\|f_{o}\right\|_{p}^{p}=n \cdot c_{0} \cdot\left\|f_{o}\right\|_{p} \cdot
$$

and thus $\left\|\hat{f}_{n}\right\|_{p, \omega} \approx n^{1 / p}$. Since $t_{k} \notin K_{n}$ for all $k \geq 1$ we also have

$$
\left\|f_{n}\right\|_{1, w}=\sum_{k=1}^{n} w^{-1}\left(t_{k}\right) \cdot\left\|M_{t_{k}} f_{0}\right\|_{1, w} \leq n^{-2} \sum_{k=1}^{n}\left\|f_{0}\right\|_{1, w}=n^{-1} \cdot\left\|f_{0}\right\|_{1, w} .
$$

Combining both estimates it is clear that we have $\left\|f_{n}\right\|_{w, \omega}^{p} \approx n^{-1 / p}$. Since the same kind of estimate gives $\left\|f_{n}\right\|_{w, \omega}^{q} \approx n^{-1 / q}$ our first estimate implies $n^{1 / q} \leq C \cdot n^{1 / p}$, which in turn implies $q \geq p$.

COROLLARY 3.6. Assume that $1 \leq p, q<\infty$, that $w$ satisfies (BD) and that $\omega \rightarrow \infty$ at infinity. Then $A_{W, \omega}^{p}(G)=A_{W, \omega}^{q}(G)$ if and only if $p=q$.

It is now important to reexamine the above proofs in order to verify that the arguments used in the proofs of Theorems 3.2 and 3.6 are in fact independent of each other. Thus we come to the following result:

THEOREM 3.7. Assume that $w_{1}, w_{2}$ satisfies (BD) and that $\omega_{i}(t) \rightarrow \infty$ for $t \rightarrow \infty$ in $\hat{G}$ for $i=1,2$. Then $A_{w_{1}, \omega_{1}}^{p_{1}}(G)=A_{w_{2}, w_{2}}^{p_{2}}$ (G) if and only if $w_{2} \approx w_{1}$ $\omega_{2} \approx \omega_{1}$ and $p_{1}=p_{2}$, i.e. if all parameters are equal.

PROOF. That these conditions are sufficient for the equality of the spaces is evident. For the converse we only have to observe that the arguments in the proof of 3.2 also work if different exponents $p_{1}$ and $p_{2}$ are involved. Thus we may conclude that corresponding weights have equivalent. Given this the equality of exponents follows from Theorem 3.6.

\section{APPROXIMATE IDENTITIES AND CONSEQUENCES.}

LEMMA 4.1. Let the weight $w$ on $G$ satisfy (BD). Then the Banach algebra $L_{w}^{1}(G)$ has a BAI whose Fourier transforms have compact support.

PROOF. Set $\Lambda_{w}^{K}=\left\{f \mid f \in L_{w}^{1}\right.$, supp $\hat{f}$ compact $\}$. Condition (BD) implies that $\Lambda_{w}^{K}$ is a dense ideal in $L_{w}^{1}(G)$, Domar [7]. Since $L_{w}^{1}(G)$ has a BAI (Reiter [2]), the proof is complete by Lemma 1.4. in Doran and Wichmann [8] .

THEOREM 4.2. If $w$ satisfies (BD), the following is true: $A_{W, \omega}^{p}(G)$ is a dense Banach ideal in $L_{W}^{1}(G)$, having an approximate identity, bounded in the norm of $L_{w}^{1}(G)$, with compactly supported Fourier transforms. In particular it is an essential Banach ideal, and $L_{w}^{1} A_{w, \omega}^{p}=A_{w, \omega}^{p} \cdot A_{w, \omega}^{p}(G)$ does not have BAI, because $G$ was assumed to be non-discrete.

PROOF. That $\mathrm{A}_{W, \omega}^{\mathrm{p}}(\mathrm{G})$ is a Banach ideal, containing $\Lambda_{W}^{\mathrm{K}}$, hence being dense in $L_{w}^{1}$, is easy to verify. In view of Lemma 4.1 it will be sufficient 
to show that any $L_{w}^{1}$-bounded AI $\left(e_{\alpha}\right)_{\alpha \in I}$ in $\Lambda_{W}^{K}$ is also an AI in $A_{W, w}^{p}$.

First we observe that boundedness of $\left(e_{\alpha}\right)_{\alpha \in I}$ in $L_{w}^{1}(G)$ implies $\sup _{\alpha}\left\|\hat{e}_{\alpha}\right\|_{\infty} \leq C<\infty$. Since $\mathscr{F L}_{W}^{1}$ is translation invariant the estimate

$$
|\hat{g}(t)|\left|1-\hat{e}_{\alpha}(t)\right| \leq\left\|\hat{g}-\hat{g} \hat{e}_{\alpha}\right\|_{\infty} \leq\left\|g-g_{*} e_{\alpha}\right\|_{1, w} \rightarrow 0 \text { (for } g \in L_{w}^{1} \text { ) }
$$

implies convergence of $\left(\hat{e}_{\alpha}(t)\right)_{\alpha \in I}$ to 1 , uniformly over compact sets. Given thus $f \in A_{W, \omega}^{p}(G)$ we choose a compact set $\hat{K} \subseteq \hat{G}$ such that $\left\|\hat{f}-\hat{f} c_{\hat{K}}\right\|_{p, \omega} \leq \varepsilon \cdot(2+2 C)^{-1}$. The local convergence to 1 implies that there is some $\alpha_{0}$ with $\left\|\hat{e}_{\alpha} c_{\hat{K}}-c_{\hat{K}}\right\|_{\infty}<\varepsilon \cdot\left(2\|\hat{f}\|_{p, \omega}\right)$ for $\alpha>\alpha_{0}$. Altogether

$$
\begin{aligned}
\left\|\hat{f}-\hat{f} \hat{e}_{\alpha}\right\|_{p, \omega} \leq & \left\|\hat{f}-\hat{f} c_{K}\right\|_{p, \omega}+\left\|\hat{f} c_{\hat{K}}-\hat{f} c_{K} \hat{\bar{e}}\right\|_{p, \omega}+\left\|\hat{f} c_{K} \hat{\bar{e}}-\hat{f} \hat{e}_{\alpha}\right\|_{p, \omega} \leq \\
& \leq\left(1+\left\|\hat{e}_{\alpha}\right\|_{\infty}\right) \cdot\left\|\hat{f}-\hat{f} c_{K}\right\|_{p, \omega}+\|\hat{f}\|_{p, \omega}\left\|\hat{e} c_{\alpha} \hat{K}-c_{\hat{K}}\right\|_{\infty} \leq \\
& <\varepsilon / 2+\varepsilon / 2=\varepsilon \text { for all } \alpha\} \alpha_{0} .
\end{aligned}
$$

Altogether it follows that $\left\|f-f_{*} e_{\alpha}\right\|_{W, \omega}^{p} \rightarrow 0$ for any $f \in A$, It is therefore obvious that $A_{w, \omega}^{p}(G)$ is an essential Banach module over $L_{w}^{1}(G)$, which in turn implies the factorization results (cf. [9]).

The unboundedness of any AI in the case of a non-discrete group (i.e. in case of non-compactness of $\hat{G}$ ) follows from the observation that a family, which converges uniformly to 1 over compact sets cannot be bounded in ${ }_{\omega}^{\mathrm{P}}(\hat{G})$ for $\mathrm{p}<\infty$, because $\omega(t) \geq \delta>0$ for all $t \in \hat{G}$ (details left to the reader).

Besides the convolution properties of $A_{W, \omega}^{p}(G)$ we also find an interesting structure of $A_{W, \omega}^{p}(G)$ with respect to pointwise multiplication. We denote by $A_{\omega}$ the Banach algebra $\mathscr{F}^{-1}\left(L_{\omega}^{1}(\hat{G})\right)$ with it's natural norm.

THEOREM 4.3. a) If $\omega$ satisfies (BD) then $A_{w, \omega}^{p}(G)$ is a dense Banach ideal in $A_{\omega}$ with respect to pointwise multiplication, containing the ideal $\mathcal{K}(G) \cap A_{\omega}$ as a dense ideal. b) Any bounded approximate identity $\left(u_{\beta}\right)_{\beta \in J}$ for $A_{\omega}$ in $\mathcal{K}(G) \cap A_{\omega}$ is also an approximate identity for $A_{W, \omega}^{p}(G)$ and $\mathcal{K}(G) \cap A_{\omega}$ is a dense subspace of $A_{W, \omega}^{p}(G)$.

PROOF. It is evident from the inversion theorem that $A_{\omega}$ is a pointwise Banach algebra, containing $\mathcal{K}(G) \cap A_{\omega}$, which is a dense ideal as a consequence of (BD). As in Lemma 4.1 one derives that $A_{\omega}$ has a BAI $\left(u_{\beta}\right)_{\beta \in J}$ in $\mathcal{K}(G) \cap A_{\omega}$, which by the arguments given in Thm.4.2. tends to the constant function 1 uniformly over compact sets. This in turn implies $\left\|u_{\beta} f-f\right\|_{1, w} \rightarrow 0$ for any $f \in L_{w}^{1}(G)$. On the other hand we have $u_{\beta}=\mathscr{F}^{-1}\left(e_{\beta}\right)$ for some BAI in $\mathrm{L}_{\omega}^{1}(\hat{G})$. Since ${ }_{\omega}^{\mathrm{p}}(\mathrm{G})$ is an essential Banach convolution module over $\mathrm{L}_{\omega}^{1}(\hat{\mathrm{G}})$ we have at the same time

$$
\left\|\left(u_{\beta} f-f\right) \hat{~}\right\|_{p, \omega}=\left\|e_{\beta} * \hat{f}-\hat{f}\right\|_{p} \rightarrow 0 \text { for } f \in A_{w, \omega}^{p}(G) \text {. }
$$

The same arguments show $\left\|u_{\beta} f-f\right\|_{W, \omega}^{p} \rightarrow$ for any BAI $\left(e_{\beta}\right)_{\beta \in J}^{W, \omega}$ in $L_{\omega}^{1}(\hat{G})$.

In order to check the density of $\mathcal{K}(\mathrm{G}) \cap A_{\omega}$ in $A_{w, \omega}(G)$ let us observe first that we can approximate $f \in A_{W, \omega}^{p}$ by functions in $\Lambda_{w}^{K}$ (in the norm of $A_{w, \omega}^{p}$ ). By the inversion theorem we see that $h$ may be assumed to be continuous. Now $u_{\beta} h$ approximates $h$, and the proof of $b$ ) is complete. 
Our next step is to check that $\mathcal{F}_{\mathrm{A}, \omega}^{\mathrm{p}}$ is a Wiener algebra in Reiter's sense ([2]) with its natural norm, given by $\hat{f} \longmapsto\|f\|_{W, \omega}^{p}$. Since we know that $A_{w}(\hat{G})$ is a Wiener algebra if $w$ satisfies (BD) (see [2]) and that

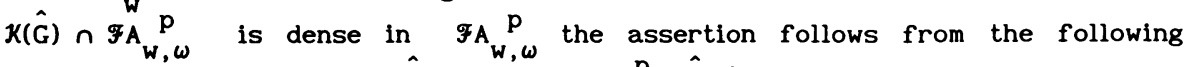
result (by choosing $A=A_{W}(\hat{G})$ and $B=\mathscr{F}_{W, \omega}^{p}(\hat{G})$ ).

LEMMA 4.4. Let $A$ be a Banach algebra of continuous functions on a lca. group G (under pointwise multiplication) which is a wiener algebra in Reiter's sense. Then any Banach ideal $\left(B,\|\|_{B}\right)$ in $A$ which contains $A \cap \mathcal{K}(G)$ as a dense subspace is again a Wiener algebra.

PROOF. The conditions to be checked will be clear from the proof:

First of all it is clear that $\left(B,\|\|_{B}\right)$ is a normed function algebra (upon renormalization, if necessary), and $h \longmapsto h(x)$ is continuous for any $x \in G, h \in B$, since $B \hookrightarrow A$ implies that $|h(x)| \leq C_{x}\|h\|_{A} \leq C_{x} \cdot C\|h\|_{B}$.

Furthermore we have to check a regularity condition (functions in $B$ separate points from open sets) and the local inversion property (given a function $f \in B$, non-vanishing over a compact set $K \subseteq G$ ) there exists $h \in B$ with $h(y)=1 / f(y)$ for all $y \in K$.

Both properties follow from the observation that 'locally' B coincides with $A$ under the given circumstances. In fact, given any compact set $K \leqslant G$ for any function $f \in A$ one can find some $f_{1} \in B$ with $f(y)=f_{1}(y)$ for all $y \in K$. This is verified as follows: By the density of $\mathcal{K}(G) \cap A$ in $A$ we can find some $g \in \mathcal{K}(G) \cap A \subseteq B$ such that $g(y) \neq 0$ for all $y \in K$. Choosing now $g_{1} \in A$ as a local inverse to $g$ over $K$ (which exists, since $A$ has local inversion) we obtain the function $g \cdot g_{1} \in(B \cap K(G)) \cdot A \subseteq B \cap K(G)$. Now it is evident that we have $f(y)=f(y) \cdot g g_{1}(y)=: f_{1}(y)$ for all $y \in G$, with $f_{1}=f \cdot g g_{1} \in A \cdot B \leq B$, as was required. Finally, the density of $\mathcal{K}(G) \cap B$ in $B$ (with the \|\|$_{B}{ }^{-n o r m}$ ) is part of the assumption, since $\mathcal{K}(G) \cap B=\mathcal{K}(G) \cap A$.

5. IDEAL THEORY AND NONFACTORIZATION IN $A_{W, \omega}^{p}(G)$.

In this section the ideal theorem for the algebras $A_{w, \omega}^{p}$ is discussed.

THEOREM 5.1. If $w$ satisfies (BD) there is a one to one correspondence between the closed ideals of ${ }_{L}^{1}{ }_{w}^{1}(G)$ and those of $A_{W, \omega}^{p}(G)$, given by the following two mappings which are inverse to each other:

(a) Given a closed ideal $J \subseteq L_{W}^{1}(G)$ the set $J \cap A_{W, w}^{p}$ is a closed ideal in $A_{w, \omega}^{p}(G)$.

(b) Given any closed ideal $I \subseteq A_{W, \omega}^{p}(G)$ the closure of $I$ in $L_{W}^{1}(G)$ is a closed ideal in $L_{W}^{1}(G)$.

PROOF. Since $A_{W, \omega}^{p}(G)$ is an essential Banach ideal in $L_{w}^{1}(G)$ (by Thm.4.2, and $L_{W}^{1}(G)$ has bounded approximate units, the result follows from the ideal theorem for abstract Segal algebras (Reiter [10], and Feichtinger [11] for detailed results of this type). 
The abstract bijection between the set of closed ideals of two spaces $A_{w, \omega_{1}}^{p}, i=1,2$, belonging to the same Beurling algebra $L_{w}^{1}(G)$, resulting from a direct application of Theorem 5.1, can be described more explicitly:

THEOREM 5.2. If $w$ satisfies (BD) for any two weights $\omega_{1}, \omega_{2}$ on $\hat{G}$ the following mappings establish a bijection between the set of closed ideals of $A_{w, \omega_{1}}^{p}(G)$ and those of $A_{w, \omega_{2}}^{p}(G)$ :

a) $\mathrm{I}_{1} \longmapsto \mathrm{I}_{1}{ }^{-} \cap A_{\mathrm{w}, \omega_{2}}^{\mathrm{p}}$ (the closure here in $\mathrm{L}_{\mathrm{w}}^{1}$ ) and

b) $I_{2} \longmapsto\left(I_{2} \cap \Lambda_{W}^{K}\right)^{-}=\left(I_{2} \cap A_{w, \omega_{1}}^{p}\right)^{-}$(the closure being taken in $A_{W, \omega_{1}}^{p}(G)$ ).

c) Moreover corresponding ideals have the same cospectrum.

PROOF. a) There is just one way to describe the composition of the two bijections as described in Thm.5.1. As for b) it is sufficient to verify that any closed ideal $I$ in an $A_{W, \omega}^{p}$-algebra can be recovered from $\operatorname{In\Lambda }_{W}^{K}$ (by taking the closure). Since the inclusion $I \subseteq\left(\operatorname{In} \Lambda_{W}^{K}\right)^{-}$is trivial it is sufficient to check that any $f \in\left(\operatorname{In} N_{W}^{K}\right)^{-}$can be approximated by elements in $I$. Thus, given $f$ and $\varepsilon>0$ we choose $g \in \Lambda_{W_{K}}^{K}$ (according to Lemma 4.1) such that $\left\|f-f_{* g}\right\|_{W, \omega}^{p}<\varepsilon$. Since $f_{* g} \in I_{*} \Lambda_{W}^{K} \subseteq I \cap \Lambda_{W}^{K}$ the result is proved.

Finally, c) follows easily from the observation that two spaces with the same $\mathrm{L}_{\mathrm{W}}{ }^{1}$-closure have the same cospectrum.

THEOREM 5.3. a) $\mathrm{A}_{\mathrm{w}_{1}, \omega_{1}}^{\mathrm{p}_{1}}(G) \subseteq \mathrm{A}_{\mathrm{W}_{2}, \omega_{2}}^{\mathrm{p}_{2}}(G)$ as a Banach ideal if and only if $w_{2} \approx w_{1}$ and $w_{1}, w_{2}$. In the positive case the bijection between ideals is established by taking the closure in the larger space, or for the inverse mapping the intersection with the smaller one (as in Thm.5.1).

PROOF. It is easy to check that the conditions are sufficient to verify the properties of a Banach ideal. Conversely the inclusion already implies that $w_{2} l w_{1}$ and $w_{2}\left\{w_{1}\right.$ by Thm.3.2. In order to prove $w_{1}\left\{w_{2}\right.$ note that we have $\quad\left\|f_{*} g\right\|_{W_{1}, \omega_{1}}^{p} \leq\|f\|_{W_{1}, \omega_{1}}^{p} \cdot\|\|_{W_{2}, w_{2}}^{p} \quad$ for $\quad f \in A_{w_{1}, \omega_{1}}^{p} \quad g \in A_{w_{2}, w_{2}}^{p}$.

Therefore

$$
\left\|L_{x}\left(f_{*} g\right)\right\|_{w_{1}, \omega_{1}}^{p} \leq\left\|f_{*}\left(L_{x} g\right)\right\|_{w_{1}, \omega_{1}}^{p} \leq\|f\|_{w_{1}, \omega_{1}}^{p} \cdot\left\|L_{x} g\right\|_{w_{2}, w_{2}}^{p} .
$$

From Lemma 2.1 the left side is equivalent to $w_{1}$ and the right side is equivalent to $w_{2}$. The above estimate then implies $w_{1}\left(w_{2}\right.$. The final statement of obvious from Theorem 5.1.

The question, whether $A_{w, \omega}^{p}(G)$ has the weak factorization property can be answered to the negative:

THEOREM 5.4. If $w$ is symmetric, i.e. if $w(x)=w(-x)$ for all $x \in G$. Then $A_{w, \omega}^{p}(G)$ does not have weak factorization (with respect to convolution).

PROOF. We deduce this result from Corollary 1.4 in [9]. We first check that $F_{A} \underset{w, w}{p}$ is a weakly self adjoint Banach algebra. This follows from the fat that the symmetry of $w$ implies that $\mathscr{F}_{\mathrm{W}}^{1}$ is closed with respect to complex conjugation. Since $\mathscr{F}_{\mathrm{A}} \underset{w}{\mathrm{p}} \omega$ is a Banach ideal in $\mathscr{F L}_{\mathrm{W}}{ }^{1}$ weak selfadjointness follows therefrom. Since by definition $\mathscr{F}_{A} \underset{w, \omega}{p}$ is contained in $\mathrm{L}_{\omega}^{\mathrm{p}}(\hat{\mathrm{G}})$, and the Haar measure on $\hat{G}$ is unbounded for non-discrete groups $G$ we 
can apply Corollary 1.4 of [9] and the proof is complete.

ACKNOWLEDGEMENTS. The major part of this work was prepared at the University of Wien. The second named author would like to thank the Department of Mathematics of the University of Vienna for its reception, and the Scientific and Technical Research Council of Turkey for financial support. He also would like to thank Doz. Hans G. Feichtinger for his hospitality and cooperation. The first named author has to carry the responsibility for the late presentation of the revised version. He wants to thank the Mathematics Department of the University of Maryland at College Park for its hospitality, as a long term visit there gave him the chance to finish the paper.

\section{References}

1. BRAUN, W. and H.G.FEICHTINGER, Banach spaces of distributions having two module structures. J.Funct. Anal. 51 (1983), (174-212).

2. REITER H. Classical Harmonic Analysis and Locally Compact Groups. Oxford University Press, Oxford, 1968.

3. FEICHTINGER, H.G. Gewichtsfunktionen auf lokalkompakten Gruppen, Sitzungsberichte der Österr. Akad.d. Wissenschaften, Mathem.-naturw. Klasse, Abteilung II, 188, Bd., 8. bis 10 (1979).

4. STEIN E.M. Singular integrals and differentiability properties of functions, Princeton University Press, Princeton, N.J., 1970.

5. GURKANLI,A.T. Some results in the weighted $A_{p}\left(\mathbb{R}^{n}\right)$ spaces, Demonstratio Mathematica Vol.19/4 (1986), 825-830.

6. FEICHTINGER,H.G. Results on Banach ideals and spaces of multipliers, Math. Scand. 41 (1977), (315-324).

7. DOMAR, Y. Harmonic analysis based on certain commutative Banach algebras, Acta Math.96 (1956), (1-66).

8. DORAN, R.S. and J.WICHMANN Approximate identity and factorization in Banach modules, Lecture Notes in Mathematics 768. Springer-Verlag, 19

9. FEICHTINGER, H.G., C.C.GRAHAM and E.H.LAKIEN Nonfactorization in commutative, weakly self-ad,joint Banach algebras, Pac.J. Math., 80 (1979), (117-125).

10. REITER H. $\underline{L}^{1}$-algebras and Segal Algebras Lecture Notes in Mathematics 231, Springer-Verlag, 1971.

11. FEICHTINGER,H.G. Zur Idealtheorie von Segal-Algebren, Manuscripta Math. 10 (1973), (307-312).

12. LARSEN,R., LIU T.S., and WANG J.K. On functions with Fourier transforms in $\mathrm{L}_{\mathrm{p}^{2}}$ Michigan Math. J. 11 (1964), (369-378).

13. MARTIN, J.C. and L.Y.H.YAP The algebra of functions with Fourier transform in $L^{p}$, Proc. Amer. Math.Soc. 24 (1970) (217-219).

14. BENEDETTO, J.J. Spectral Synthesis, Academic Press Inc., 1975.

15. BURNHAM, J.T. Closed ideals in subalgebras of Banach algebras I, Proc. Amer.Math.Soc. 32 (1972), (551-555).

16. FEICHTINGER,H.G. Compactness in translation invariant Banach spaces of distributions and compact multipliers, J. Math. Anal.Appl. 102 (1984), (289-327).

17. HEWITT,E. and K.A.ROSS. Abstract Harmonic Analysis, II. Grundl.math. Wiss., Springer Verlag, 1963. 


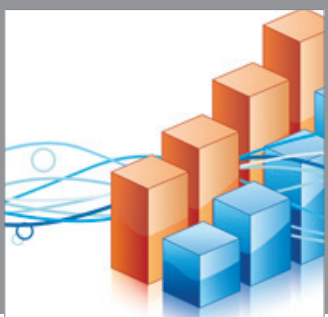

Advances in

Operations Research

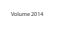

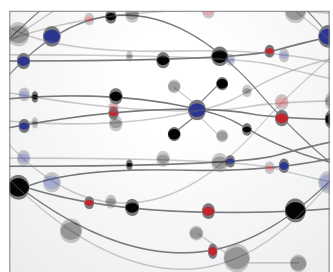

\section{The Scientific} World Journal
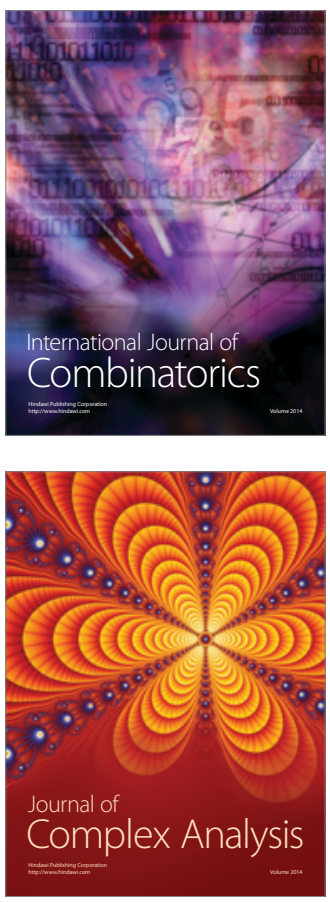

International Journal of

Mathematics and

Mathematical

Sciences
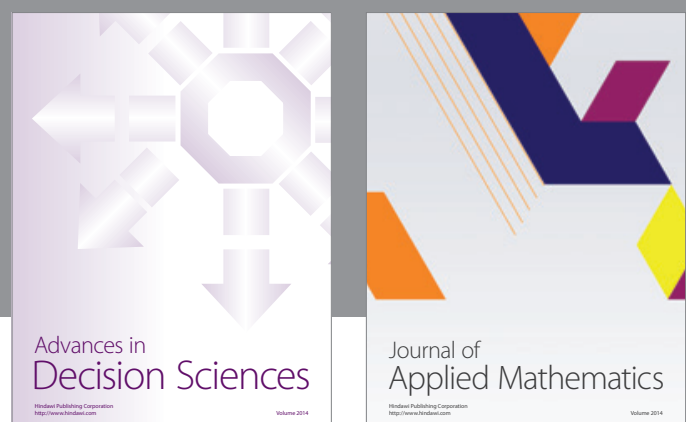

Journal of

Applied Mathematics
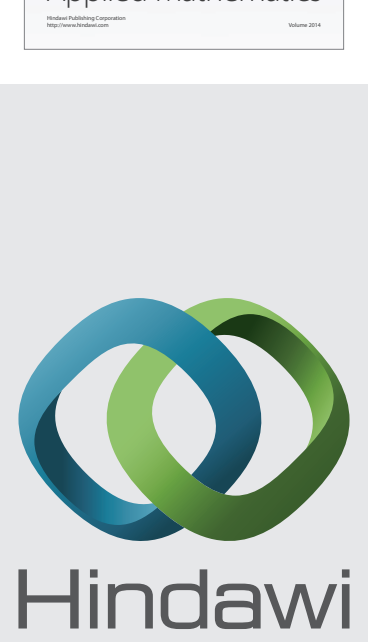

Submit your manuscripts at http://www.hindawi.com
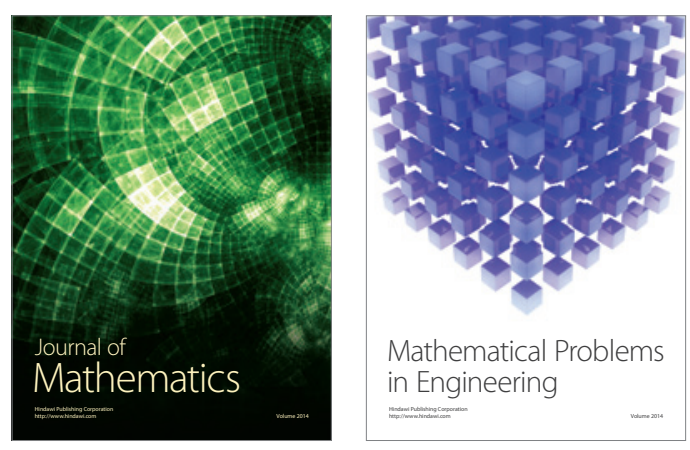

Mathematical Problems in Engineering
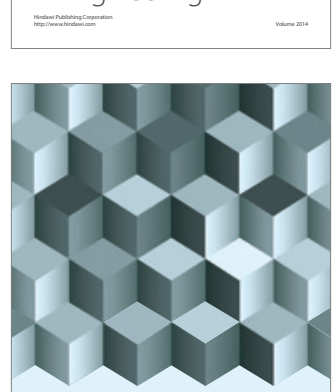

Journal of

Function Spaces
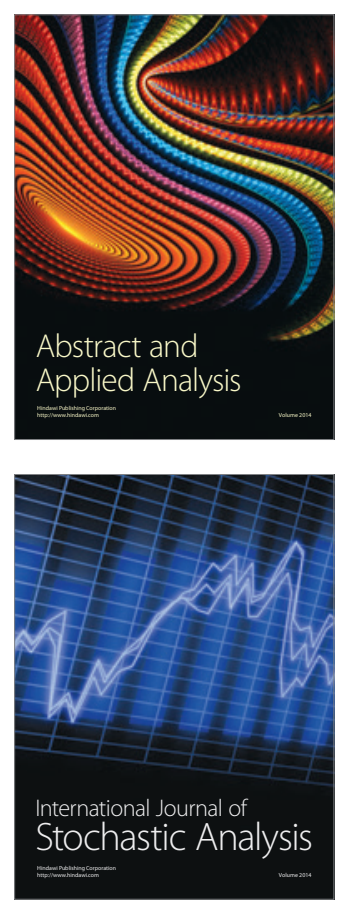

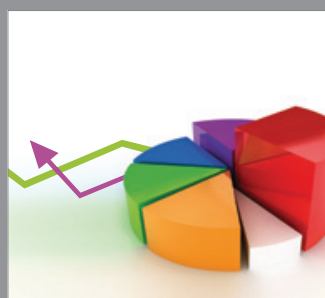

ournal of

Probability and Statistics

Promensencen
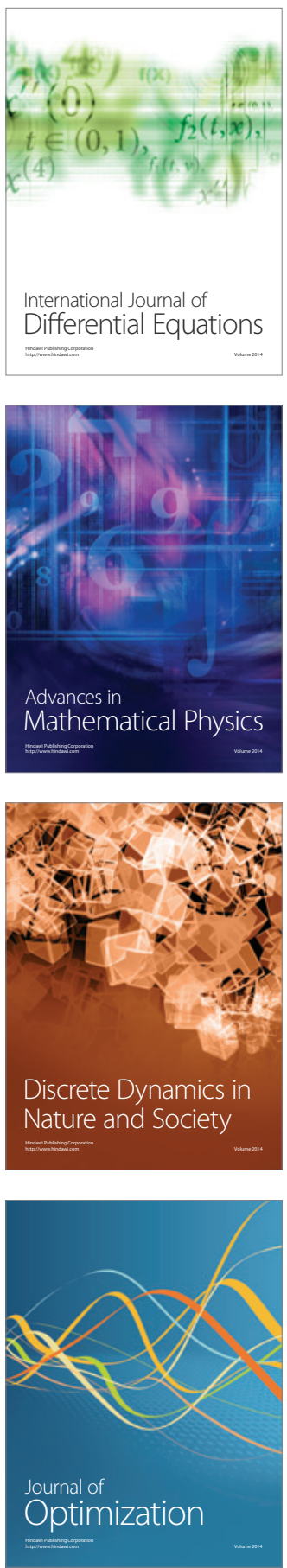